\title{
Acute toxicity and oxidative damage induced by silica nanorattle in vivo
}

\author{
FU ChangHui ${ }^{1,2}$, LIU TianLong ${ }^{2}$, TANG FangQiong ${ }^{2 *}$, CHEN Dong $^{2}$, LI LinLin $^{2}$, \\ LIU HuiYu ${ }^{2} \&$ LI XiaoMin ${ }^{1 *}$ \\ ${ }^{1}$ School of Animal Science and Technology, Southwest University, Chongqing 400715, China; \\ ${ }^{2}$ Laboratory of Controllable Preparation and Application of Nanomaterials, Technical Institute of Physics and Chemistry, Chinese Academy of \\ Sciences, Beijing 100190, China
}

Received December 14, 2011; accepted February 23, 2012; published online May 6, 2012

\begin{abstract}
Hollow mesoporous nanomaterial is a kind of promising new drug delivery system due to their unique hollow structures. In order to evaluate the toxicity of silica nanorattle $(\mathrm{SN})$ particles in vivo, 40 female mice were used in this study to investigate the acute toxicity and oxidative damage. Mice were intravenously injected with SN suspension in sterile 5\% glucose at 40, $80 \mathrm{and} 240 \mathrm{mg} / \mathrm{kg}$, respectively. The control group was administrated with equal-volume 5\% glucose. Weight, feed intake, hematology analysis, blood biochemical assay and histopathology diagnosis were examined. The activities of SOD, GSH and CAT were measured as well. The results demonstrated that the levels of ALT and AST in the mice treated with $240 \mathrm{mg} / \mathrm{kg} \mathrm{SN}$ increased significantly as compared with the control group $(P<0.05)$, whereas the contents of BUN and CREA changed unremarkably. The activity of SOD induced by $\mathrm{SN}$ in the liver decreased significantly $(P<0.05)$. In summary, this study revealed that liver was the target organ of the SN. It also can be concluded that activity of SOD played an important role in liver injury caused by SN.
\end{abstract}

silica nanorattle, acute toxicity, oxidative stress, superoxide dismutase

Citation: $\quad$ Fu C H, Liu T L, Tang F Q, et al. Acute toxicity and oxidative damage induced by silica nanorattle in vivo. Chin Sci Bull, 2012, 57: 2525-2532, doi: 10.1007/s11434-012-5187-y

In the past few years, mesoporous nanomaterials have attracted more and more attention owing to their great potential application in many fields, such as physics, chemistry and textile, especially in biomedicine [1-5]. Mesoporous silica has been extensively explored as drug carriers due to their unique mesoporous structures and large surface area [6]. However, the drug storage capacity of the conventional mesoporous materials is relatively low, and the uneven morphology of them is not optimal for drug delivery. To overcome these problems, one strategy is to synthesize mesoporous hollow silica nanoparticles with penetrating pore channels from outside to the inner hollow capacity [7]. However, for synthesizing nanomaterials with hollow structure, the fabrication processes are often so tedious that they are difficult to be scaled up. Recently, we reported a flexible, scalable and robust method to prepare rattle-type mesopo-

*Corresponding authors (email: tangfq@mail.ipc.ac.cn; lixiaomin662@sina.com) rous silica hollow spheres. Compared to conventional methods used for preparing rattle-type nanomaterials, our selective etching strategy has many obvious advantages: it is a scalable and general method to fabricate functional nanoparticles with significant monodispersion [1]. The silica nanorattle with special structures provides a large interstitial hollow space to achieve high drug loading amount and multistage drug release, suggesting potential applications in the fields of targeted tumor therapy and imaging in vivo [4,8,9].

With the increasing application of mesoporous hollow nanoparticles in biomedicine, the concerns on the biosafty aspect caused by mesoporous nanoparticles are also increasing [10-12]. So far, most of the researches were focused on in vitro study [13]. Only a few in vivo studies have been concentrated on the toxicity in respiratory system [14]. With the rapid development of mesoporous silica in the biomedical application, especially as drug carriers, mesoporous silica may enter the body through intravenous injec- 
tion, subcutaneous injection and so on $[8,15]$. However, the biological effect and biodistribution caused by the mesoporous silica particles exposed through these routes have not been sufficiently understood.

In this paper, the toxicity of silica nanorattle $(\mathrm{SN})$ particles in vivo after intravenous administration was systematically evaluated. The potential roles of oxidative stress in the damage caused by SN were also investigated.

\section{Materials and methods}

\subsection{Materials}

SN was provided by Laboratory of Controllable Preparation and Application of Nanomaterials, Technical Institute of Physics and Chemistry, Chinese Academy of Sciences [1]. Ethanol and glucose was purchased from Beijing Chemical Works. Formaldehyde solution were obtained from West Long Chemical Indrustries Co., Ltd. Paraffin (melting point, $50-52^{\circ} \mathrm{C}$ and $52-54^{\circ} \mathrm{C}$ ) were obtained from Shanghai chemical Industries Co., Ltd. Paraplast ${ }^{\circledR}$ embedding media was purchased from sigma (Sigma-Aldrich, St.Louis, MO, USA). Glutathione (GSH), malondialdehyde (MDA), total antioxidant capacity (T-AOC), catalase (CAT), inducible nitric oxide synthase (iNOS) and superoxide dismutase (SOD) assay kits were provided by Nan Jing Jian Cheng Bioengineering Institute.

\subsection{Preparation and characterization of SN}

SN was soaked in $75 \%$ ethanol for $4 \mathrm{~h}$ and washed with sterile ultrapure water for 3 times. Then they were dispersed in sterile $5 \%$ glucose solution. Lastly, $\mathrm{SN}$ suspension was sterilized by UV irradiation for $30 \mathrm{~min}$ and sonicated for $15 \mathrm{~min}$ before use. The hydrodynamic diameter and Zeta potential of $\mathrm{SN}$ in the solution $(1 \mathrm{mg} / \mathrm{mL})$ were investigated at $25^{\circ} \mathrm{C}$ with Zetasizer $3000 \mathrm{HS}$ (Malvern). Morphology and structure of SN were observed with JEOL-200CX transmission electron microscope (TEM).

\subsection{Animals treatments}

All animal experiments were preformed in compliance with the local ethics committee. Forty female ICR mice (obtained from Vital River Laboratory Animal Technology Co. Ltd.), aged 6-8 weeks and weighted 20-22 g, were used in the study. Mice were randomly divided into four groups ( $n=$ 10 for each group). The animals were intravenously injected with SN suspension in sterile 5\% glucose at 40, 80 and 240 $\mathrm{mg} / \mathrm{kg}$, respectively. Mice in control group were injected with equal-volume $5 \%$ glucose.

\subsection{Clinical observation}

Clinical manifestations of the mice were observed intimately after dosing SN suspension at different levels. After intravenous injection, mortality, animal feed intake and body weight were recorded every day.

\subsection{Hematology analysis and serum biochemical assays}

After injection for $14 \mathrm{~d}$, blood was drawn from orbital angular vein blood $(0.1 \mathrm{~mL})$ with potassium EDTA collection tube for hematology analysis. Blood samples, which were collected via the ocular vein (about $0.8-1.0 \mathrm{~mL}$ each mouse), were centrifuged at $3000 \mathrm{r} / \mathrm{min}$ for $20 \mathrm{~min}$ in order to separate serum.

\subsection{Histopathological assays}

Mice were sacrificed at $14 \mathrm{~d}$ after administration, and the following organs were collected: liver, spleen, lung, kidney, heart and brain. Then these tissues were fixed in $10 \%$ neutral formalin, embedded in paraffin, sectioned and stained with hematoxylin and eosin (HE) for histological examination using standard techniques. The slides were observed, and photos were taken using an optical microscope (Nikon Eclipese Ti-S).

\subsection{Oxidative damage analysis}

After mice had been sacrificed, a portion of liver was frozen for detecting oxidative damage indicators. SOD, MDA, T-AOC, CAT, iNOS and GSH were tested according to the product instructions.

\subsection{Statistics analysis}

Results were expressed as mean \pm standard deviation $(\bar{x} \pm s)$. Multigroup comparisons of the means were carried out by one-way analysis of variance ANOVA test using SPSS 17.0 software. $P<0.05$ was considered statistically significant.

\section{Results}

\subsection{Characterization of SN}

TEM image and size distribution of SN suspension in sterile $5 \%$ glucose are shown in Figure 1. The well-dispersed SN possessed an obvious hollow structure with a movable core. The average diameter of the SN from TEM image was determined to be $110 \pm 10 \mathrm{~nm}$. And the hydrodynamic diameter of the $\mathrm{SN}$ in sterile $5 \%$ glucose was $180 \pm 55 \mathrm{~nm}$, as shown in Figure 1(b). The SN had a positive $\xi$-potential of about $37.2 \pm 1.48 \mathrm{mV}$.

\subsection{Clinical observation}

In the period of experiments, no distinctive changes in general 

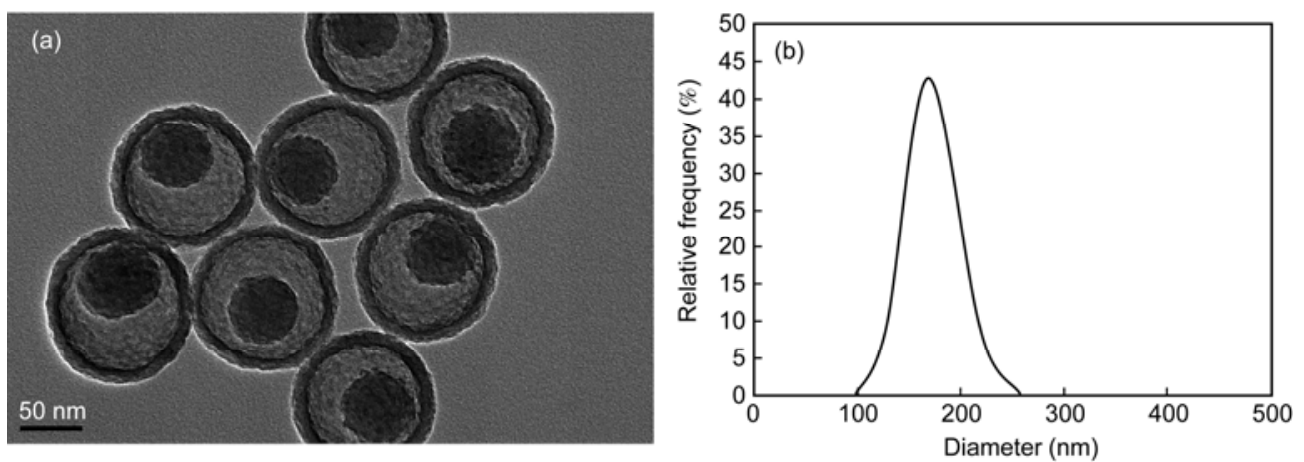

Figure 1 TEM image (a) and the size distribution (b) of SN in sterile $5 \%$ glucose. Scale bar is $50 \mathrm{~nm}$.

behavior involving feed intake, water-drinking, reflection action, respiration and body temperature were observed in the experimental groups compared with the control. No deaths were observed in the treatment groups. The average daily feed intake (ADFI) and body weight of the treated mice showed insignificant changes as compared with the control group $(P>0.05)$, as revealed in Figure 2.

\subsection{Hematology analysis}

Representative hematology markers were analyzed, including red blood cell count (RBC), hemoglobin (HGB), hematocrit (HCT), mean corpuscular hemoglobin concentration (MCHC), mean corpuscular volume (MCV), mean corpuscular hemoglobin $(\mathrm{MCH})$, platelet count (PLT) and white blood cell count (WBC). In contrast to the control group, all hematology makers were not significantly affected after administration of $\mathrm{SN}$ at 40,80 and $240 \mathrm{mg} / \mathrm{kg}(P>0.05)$ (Figure 3).

\subsection{Serum biochemical assay}

Representative serum biochemical indicators involving alanine aminotransferase (ALT), aspartate aminotransferase (AST), urea nitrogen (BUN) and creatinine (CREA) were detected as well. The contents of serum ALT or AST were significantly increased with SN administration at $240 \mathrm{mg} / \mathrm{kg}$, while no significant increase was observed with that at 40 and $80 \mathrm{mg} / \mathrm{kg}$ as compared with the control group $(P<0.05)$ (Figure 4(a),(b)). However, BUN and CREA level changed unremarkably after injecting $\mathrm{SN}$ at 40,80 and $240 \mathrm{mg} / \mathrm{kg}$ (Figure 4(c),(d)).

\subsection{Histopathological examinations}

The paraffin slices of representative organs including liver, spleen, lung, kidney, heart and brain were prepared. And the morphology changes of these organs were analyzed. Compared with the control group, there were no apparent histopathological abnormalities or lesions. The cell nucleus was bright and the cytoplasm was abundant for the groups treated with SN at 40 and $80 \mathrm{mg} / \mathrm{kg}$ (Figure 5(b)). But lymphocytic infiltration and swelling in liver were obviously observed at the group after administration at $240 \mathrm{mg} / \mathrm{kg}$, as shown in Figure 5(c). Spleen samples obtained from the treatment groups showed no significant changes, and the size of the red pulp was well-defined as compared with that of the control group (Figure 5(e), (f)). Kidney, heart, lung, and brain samples also showed no remarkable change in the morphology, as revealed in Figure 5(g)-(i) and Figure 6(a)-(i).

\subsection{Oxidative damage}

Oxidative stress is considered to be one of the key mecha-
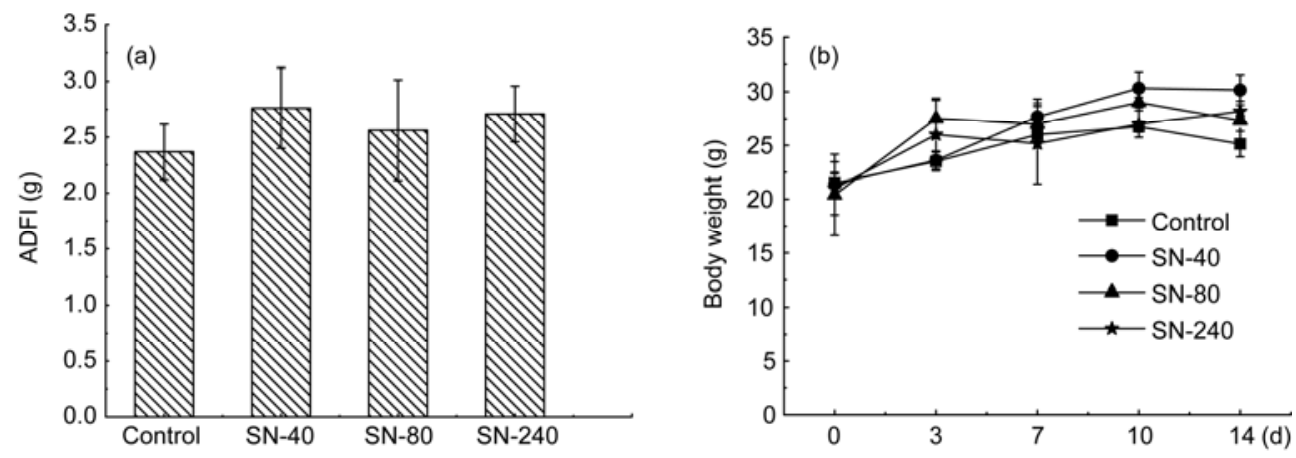

Figure 2 The change of average daily feed intake (a) and body weight (b) obtained from mice injected at different doses of SN. SN-40, SN-80 and SN-240 represent the dose of $\mathrm{SN}$ at 40,80 and $240 \mathrm{mg} / \mathrm{kg}$, respectively. 

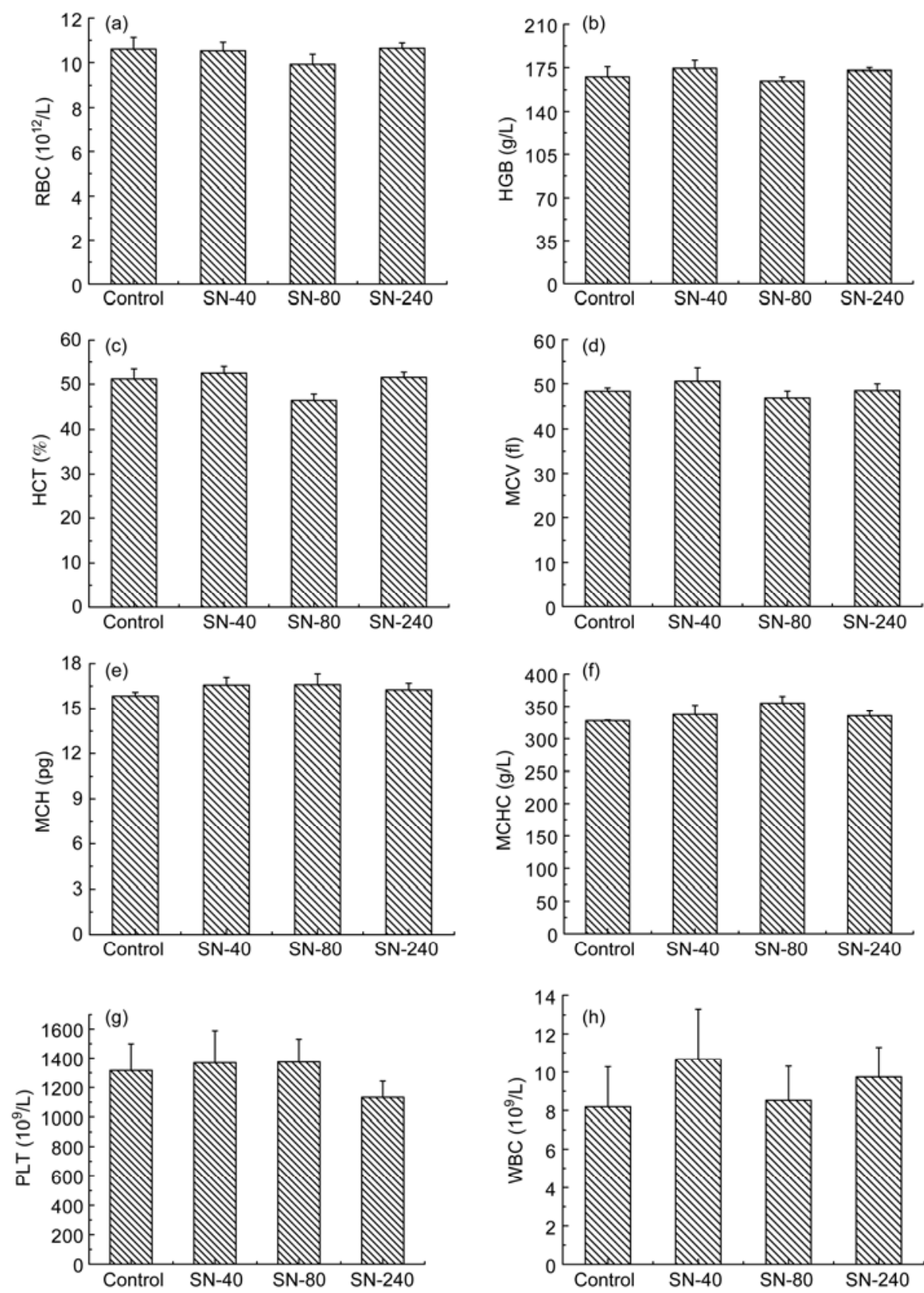

Figure 3 Effect of different doses of SN on hematology at 14 d. (a)-(h) are red blood cell count (RBC), hemoglobin (HGB), hematocrit (HCT), mean corpuscular volume (MCV), mean corpuscular hemoglobin $(\mathrm{MCH})$, mean corpuscular hemoglobin concentration (MCHC), platelet count (PLT) and white blood cell count (WBC), respectively.

nisms for the toxicity of nanomaterials. In this paper, antioxidative indicators of the liver were detected. The results are shown in Figure 7. It can be observed that the activity of SOD in the treatment groups decreased significantly $(P<0.05)$. However, there was no significant difference between that of the three groups treated with SN, as summarized in Figure 7(a). The content of GSH and MDA showed no significant variation in the groups treated with $\mathrm{SN}$ at 40, 80 and $240 \mathrm{mg} / \mathrm{kg}$ as well (Figure 7(b), (e)). The activity of CAT had a trend to increase in the treatment groups, but it was not significant $(P>0.05)$. And the activity of iNOS in the group dosed at $80 \mathrm{mg} / \mathrm{kg}$ was also slightly elevated. Nevertheless, iNOS of the groups treated at 40 and $240 \mathrm{mg} / \mathrm{kg}$ decreased instead. But the change was not significant $(P>0.05)$, as shown in Figure 7(f).

\section{Discussion}

Very recently, several studies have demonstrated that SN particles not only can serve as promising drug carriers for high tumor targeting efficiency and therapy efficacy, but 

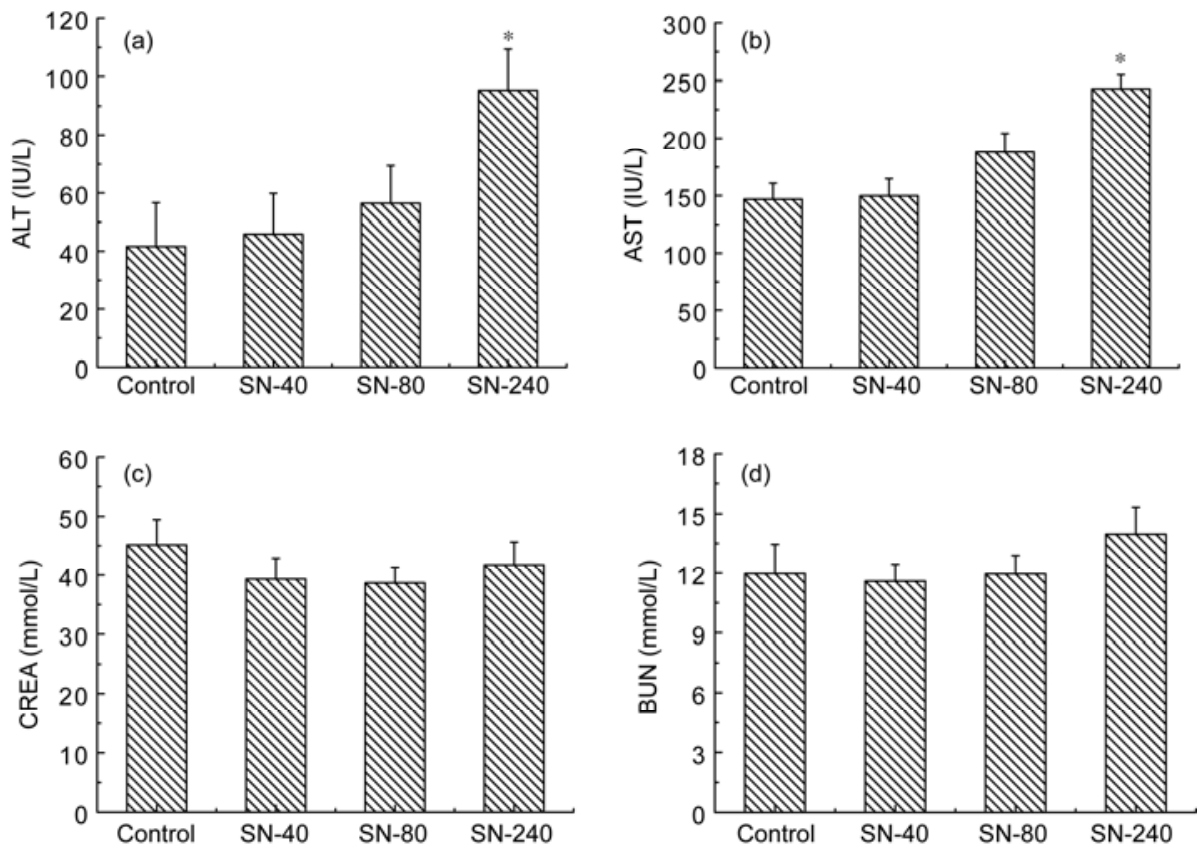

Figure 4 Effect of different doses of SN on serum biochemistry at $14 \mathrm{~d}$. (a)-(d) are alanine aminotransferase (ALT), aspartate aminotranferase (AST), creatinine (CREA) and blood urea nitrogen (BUN), separately. * denotes statistical significance for the comparison of control, $P<0.05$.
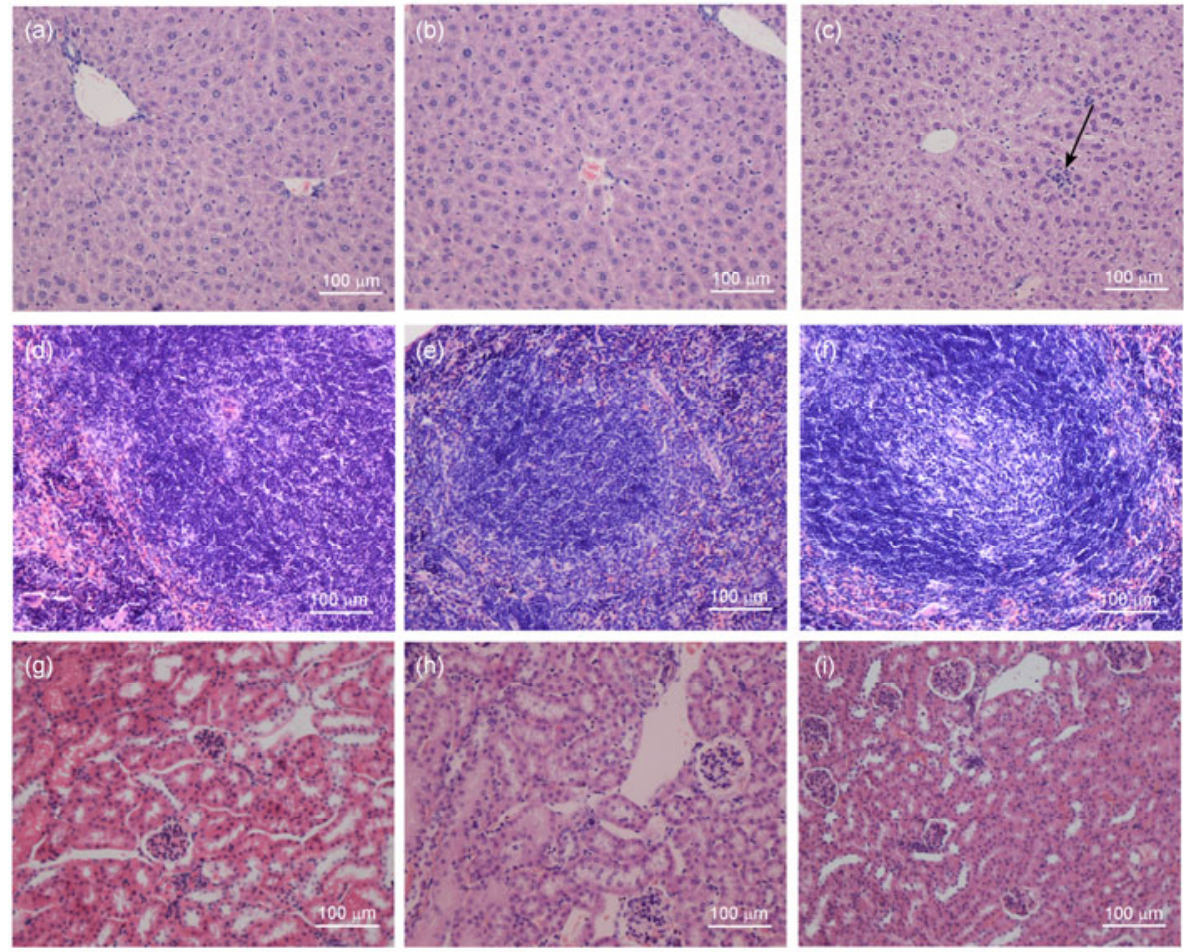

Figure 5 Histological analyses of liver ((a)-(c)), spleen ((d)-(f)) and kidney ((g)-(i)) from mice injected with SN at control group ((a), (d), (g)), $40 \mathrm{mg} / \mathrm{kg}$ ((b), (e), (h)) and $240 \mathrm{mg} / \mathrm{kg}$ ((c), (f), (i)), arrows indicates the infiltration. Scale bar is $100 \mu \mathrm{m}$.

also for reducing the systematic toxicity [16,17]. However, the biological safety effect is sill being a pendent question of SN particles $[18,19]$. Therefore, the toxicity of SN in vivo exposed by intravenous injection was evaluated in this study.
We found that exposure of $\mathrm{SN}$ at 40 and $80 \mathrm{mg} / \mathrm{kg}$ would not affect the value of hematologic markers, serum biochemical markers compared with the control group, which indicates that $\mathrm{SN}$ would not induce evident adverse effects 

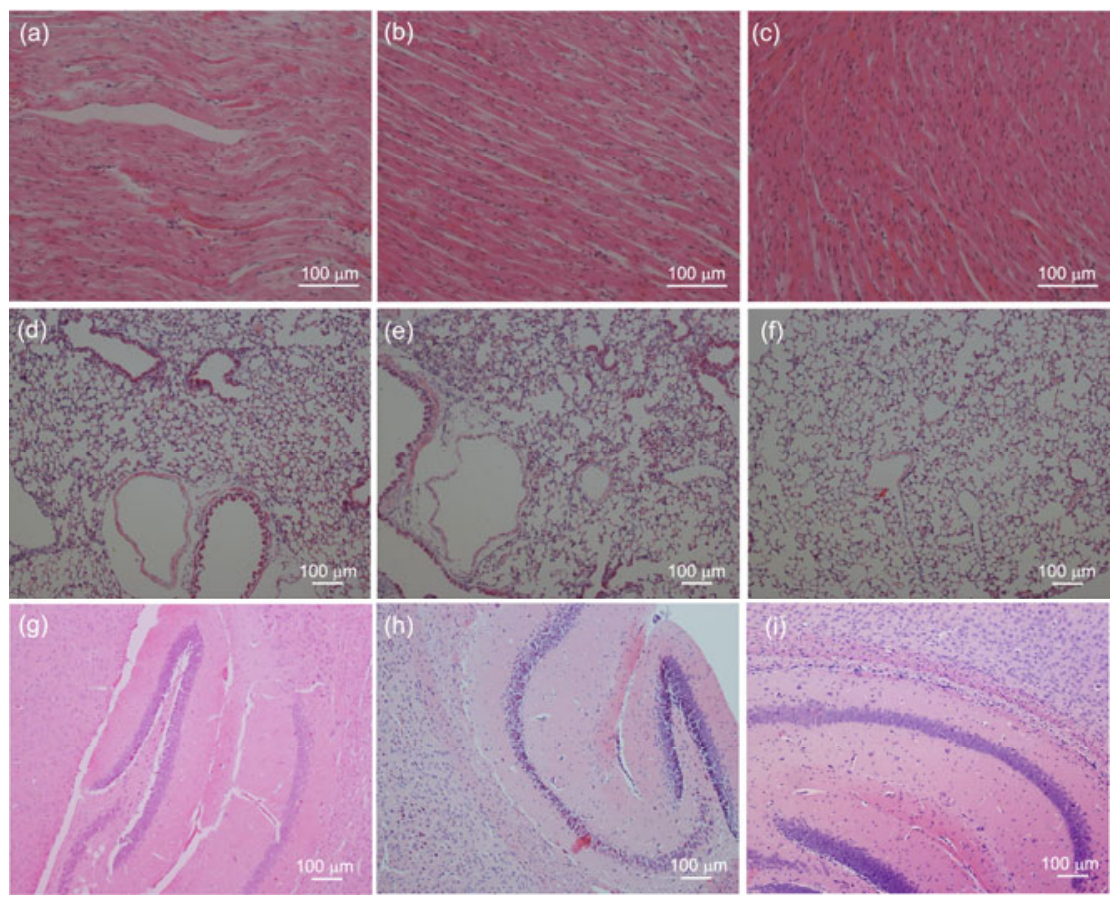

Figure 6 Histological analyses of heart ((a)-(c)), lung ((d)-(f)) and brain ((g)-(i)) from mice injected with SN at control group ((a), (d), (g)), $40 \mathrm{mg} / \mathrm{kg}((\mathrm{b})$, (e), (h)) and $240 \mathrm{mg} / \mathrm{kg}((\mathrm{c}),(\mathrm{f})$, (i)). Scale bar is $100 \mu \mathrm{m}$.
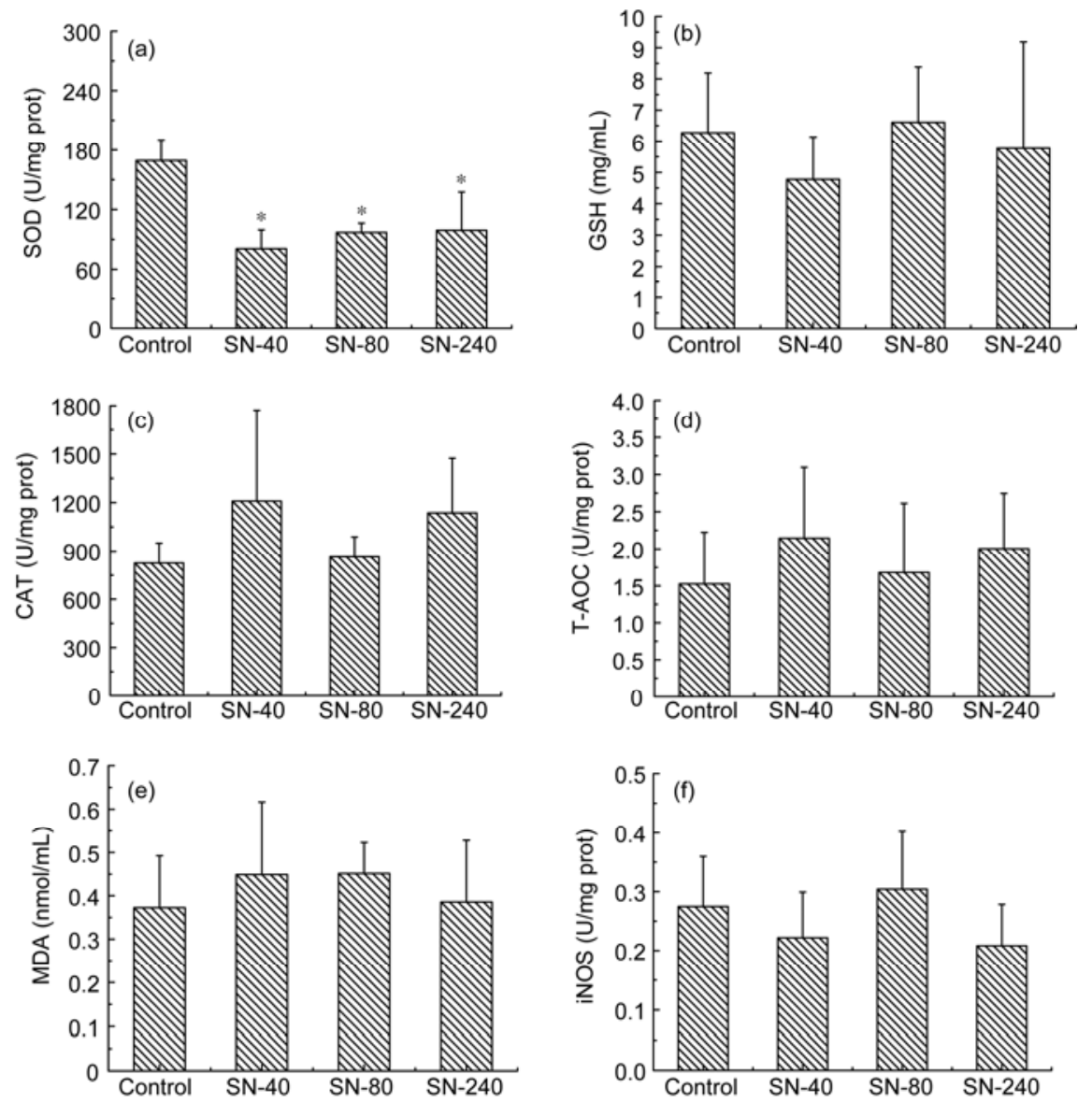

Figure 7 The change of oxidative stress induced by different doses of SN through tail intravenous injection. * denotes statistical significance in comparison with control, $P<0.05$. (a)-(f) are superoxide dismutase (SOD), glutathione (GSH), catalase (CAT), total antioxidant capacity (T-AOC), malondialdehyde (MDA) and inducible nitric oxide synthase (iNOS), respectively. 
at dose of 40 and $80 \mathrm{mg} / \mathrm{kg}$.

The obvious elevation of blood biochemical indexes and the significant variation in the histopathology of liver tissue were observed at $240 \mathrm{mg} / \mathrm{kg}$ dose. The predominant increase in ALT and AST of the mice was caused by the injection of high dose of SN. ALT and AST are important aminotransferases in the body. ALT is mainly distributed in the liver, kidney and heart and so on. And the highest concentration of AST is found in heart, liver, skeleton muscle and kidney [20]. Both ALT and AST can be released into the blood when these tissues are injured. As a result, the contents of ALT and AST in the blood would increase significantly. Thus, the changes of ALT and ASL in blood offer important references as probes of damages to the heart and liver [21,22]. We found that administration of high-dose SN obviously increased ALT and AST contents but would not cause any abnormalities of heart. It caused inflammation to the liver, which means the increase of transaminase indicators are caused by liver injury. In addition, the histomorphology studies suggest that injection of SN at $240 \mathrm{mg} / \mathrm{kg}$ would not cause damages to spleen, kidney, lung and brain. Thus, liver is to be the major organ for toxicity by the intravenous administration of the SN particles.

Oxidative damage to the cells and tissues induced by nanomaterials has attracted broad attention. It is considered to be one of the key mechanisms in the toxicity of nanomaterials [23]. Oxidative damage is the result of unbalance between the oxidant and antioxidant processes. Antioxidant defense system plays an important role in the elimination of oxygen radical in vivo, which includes enzymatic scavengers (SOD, CAT and GPX) and some small-molecule antioxidants (GSH, vitamin C and vitamin E) [24]. Here, we investigated the antioxidant defense system involving the index of SOD, CAT, GSH, and so on. The results showed that the activity of SOD was decreased after intravenous injection of SN, and no significant changes of other indicators were detected in mice hepatocytes. It may be related to the important role of SOD in the antioxidant system in vivo, because SOD is the unique antioxidant enzyme for scavenging superoxide anion in the body [25]. It has been suggested that SN could trigger the generation of reactive oxygen species (ROS), which results in injuries of the tissues [26]. A large amount of SOD was consumed to diminish ROS level, which results in disturbance of the balance between the oxidant and antioxidant process [27]. Thus, this study reminds us that the damages to the liver may be related to the decreased activity of SOD caused by SN. It offers us an important basis for further investigating the toxicity mechanism of nanomaterials and constructing the corresponding strategies for preventions.

From the above results, no apparent toxic effects were observed at low dose of $\mathrm{SN}$, whereas injection of $\mathrm{SN}$ at high dose would cause some damages to the liver of mice. Therefore, through adjusting the dose of drug carriers, it can not only obtain a satisfactory therapeutic efficacy, but also avoid the toxic effects caused by the carriers. It provides us important experimental information for the clinical application of SN as nano-drug carriers.

\section{Conclusion}

In conclusion, we demonstrate that the as-prepared $\mathrm{SN}$ has good biocompatibility, which provides important foundation of toxicity research for the exploitation of SN as new drug carriers. SN would induce liver damage when exposed at high concentration. The injury may be related to the decreased activity of SOD. The results offer us useful information for the further research on the toxicity mechanism caused by nanoparticles.

This work was supported by the National Natural Science Foundation of China (30900349 and 81171454).

1 Chen D, Li L L, Tang F Q, et al. Facile and scalable synthesis of tailored silica "Nanorattle" structures. Adv Mater, 2009, 21: 3804-3807

2 Chen D, Tan L F, Liu H Y, et al. Fabricating superhydrophilic wool fabrics. Langmuir, 2010, 26: 4675-4679

3 Du X, He J H. Spherical silica micro/nanomaterials with hierarchical structures: Synthesis and applications. Nanoscale, 2011, 3: 3984-4002

4 Liu H Y, Chen D, Li L L, et al. Multifunctional gold nanoshells on silica nanorattles: A platform for the combination of photothermal therapy and chemotherapy with low systemic toxicity. Angew Chem Int Ed, 2011, 50: 891-895

5 Shen D H, Wang X P. The Process of standardization of nanotechnology and related ethical issues (in Chinese). Chin Sci Bull (Chin Ver), 2011, 56: 131-134

6 Vallet-Regi M, Ramila A, del Real R P, et al. A new property of MCM-41: Drug delivery system. Chem Mater, 2001, 13: 308-311

7 Lou X W, Archer L A, Yang Z C. Hollow micro-/nanostructures: Synthesis and applications. Adv Mater, 2008, 20: 3987-4019

8 Li L L, Guan Y Q, Liu H Y, et al. Silica nanorattle-Doxorubicinanchored mesenchymal stem cells for tumor-tropic therapy. ACS Nano, 2011, 5: 7462-7470

9 Li L L, Tang F Q, Liu H Y, et al. In vivo delivery of silica nanorattle encapsulated Docetaxel for liver cancer therapy with low toxicity and high efficacy. ACS Nano, 2010, 4: 6874-6882

10 Maynard A D, Aitken R J, Butz T, et al. Safe handling of nanotechnology. Nature, 2006, 444: 267-269

11 Liu Y, Chen C Y. Safety and risk assessment of nanomaterials (in Chinese). Chin Sci Bull (Chin Ver), 2011, 56: 119-125

12 Chang X L, Zhu Y, Zhao Y L. Size and structure effects in the nanotoxic response of nanomaterials (in Chinese). Chin Sci Bull (Chin Ver), 2011, 56: 108-118

13 Malugin A, Herd $H$, Ghandehari $H$. Differential toxicity of amorphous silica nanoparticles toward phagocytic and epithelial cells. J Nanopart Res, 2011, 13: 5381-5396

14 Chen Z, Meng H, Xing G M, et al. Age-related differences in pulmonary and cardiovascular responses to $\mathrm{SiO}_{2}$ nanoparticle inhalation: Nanotoxicity has susceptible population. Environ Sci Technol, 2008, 42: 8985-8992

15 Gordon S, Teichmann E, Young $\mathrm{K}$, et al. In vitro and in vivo investigation of thermosensitive chitosan hydrogels containing silica nanoparticles for vaccine delivery. Eur J Pharm Sci, 2010, 41: 360-368

16 Meng H, Xue M, Xia T, et al. Use of size and a copolymer design feature to improve the biodistribution and the enhanced permeability and retention effect of Doxorubicin-loaded mesoporous silica nanoparticles in a murine xenograft tumor model. ACS Nano, 2011, 5: 
4131-4144

17 Gao Y, Chen Y, Ji X F, et al. Controlled intracellular release of Doxorubicin in multidrug-resistant cancer cells by tuning the shellpore sizes of mesoporous silica nanoparticles. ACS Nano, 2011, 5: 9788-9798

18 Liu T L, Li L L, Fu C H, et al. Pathological mechanisms of liver injury caused by continuous intraperitoneal injection of silica nanoparticles. Biomaterials, 2012, 33: 2399-2407

19 Liu T L, Li L L, Teng X, et al. Single and repeated dose toxicity of mesoporous hollow silica nanoparticles in intravenously exposed mice. Biomaterials, 2011, 32: 1657-1668

20 Finsterer J, Mittendorfer B, Neuhuber W, et al. Influence of disposable, concentric needle electrodes on muscle enzyme and lactate serum levels. J Electromyogr Kines, 2002, 12: 329-337

21 Giannini E, Botta F, Testa E, et al. The 1-year and 3-month prognostic utility of the AST/ALT ratio and model for end-stage liver disease score in patients with viral liver cirrhosis. Am J Gastroenterol, 2002, 97: 2855-2860

22 Shteyer E, Yatsiv I, Sharkia M, et al. Serum transaminases as a prognostic factor in children post cardiac surgery. Pediatr Int, 2011, 53: $725-728$

23 Xia T, Kovochich M, Brant J, et al. Comparison of the abilities of ambient and manufactured nanoparticles to induce cellular toxicity according to an oxidative stress paradigm. Nano Lett, 2006, 6: 1794-1807

24 Olsvik P A, Kristensen T, Waagb $\varnothing$ R, et al. mRNA expression of antioxidant enzymes (SOD, CAT and GSH-Px) and lipid peroxidative stress in liver of Atlantic salmon (Salmo salar) exposed to hyperoxic water during smoltification. Comp Biochem Physiol C Toxicol Pharmacol, 2005, 141: 314-323

25 Scandalios J G. Oxygen stress and superoxide dismutases. Plant Physiol, 1993, 101: 7-12

26 Park E J, Park K. Oxidative stress and pro-inflammatory responses induced by silica nanoparticles in vivo and in vitro. Toxicol Lett, 2009, 184: 18-25

27 Liu S, Hou W, Yao P, et al. Quercetin protects against ethanolinduced oxidative damage in rat primary hepatocytes. Toxicol in Vitro, 2010, 24: 516-522

Open Access This article is distributed under the terms of the Creative Commons Attribution License which permits any use, distribution, and reproduction in any medium, provided the original author(s) and source are credited. 